\title{
Distillation optimization: Parameterized relationship between feed flow rate of a steady-state distillation column and heat duties of reboiler and condenser
}

\author{
Ivan Sukin ${ }^{1}$, Anatoly Tsirlin ${ }^{1}$, Alexander Balunov², and Ilya Starodumov ${ }^{3}$ \\ ${ }^{1}$ Ailamazyan Program Systems Institute of Russian Academy of Sciences \\ ${ }^{2}$ Yaroslavl State Technical University \\ ${ }^{3}$ Ural Federal University named after the first President of Russia B N Yeltsin
}

June 5, 2021

\begin{abstract}
The paper considers the problem of maximum efficiency for the system of distillation columns. Columns in such systems are connected in parallel or sequential way. The mixture being separated is assumed to be close to ideal one. Authors parameterize the relationship between feed flow rate and heat duties of a steady-state binary distillation column using two parameters: the reversible efficiency and the irreversibility coefficient. This relationship is later being used to solve the problems about optimal distribution of heat and feed flows within the system. The results obtained allow to estimate minimum heat energy demand for distillation of the given feed flow, maximum performance and efficiency of the system.
\end{abstract}

\section{Hosted file}

Opt_SIST-M-en.pdf available at https://authorea.com/users/418138/articles/525001distillation-optimization-parameterized-relationship-between-feed-flow-rate-of-a-steadystate-distillation-column-and-heat-duties-of-reboiler-and-condenser 
figures/pic1new1/pic1new1-eps-converted-to.pdf 
figures/opt1/opt1-eps-converted-to.pdf 
figures/opt2/opt2-eps-converted-to.pdf 
figures/opt3/opt3-eps-converted-to.pdf 